\title{
Two-step heating synthesis of sub-3 millimeter-sized orthorhombic black phosphorus single crystal by chemical vapor transport reaction method
}

\author{
Ziming Zhang ${ }^{1 \dagger}$, Xin Xin ${ }^{2,3 \dagger}$, Qingfeng Yan $^{1 *}$, Qiang Li $^{1}$, Yi Yang ${ }^{2,3}$ and Tian-Ling Ren ${ }^{2,3^{*}}$
}

\begin{abstract}
A facile and green strategy to synthesize orthorhombic black phosphorus (o-BP) single crystals with high yield $(\sim 90 \%)$ and large size (sub-3 millimeter) is presented. The strategy was based on a two-step heating chemical vapor transport (CVT) reaction method, in which tin and iodine $\left(\mathrm{Sn} / \mathrm{I}_{2}\right)$ was used as mineralization additives and red phosphorus as precursor. Tin phosphide was the only by-product captured at the end of reaction, which greatly simplified the subsequent separation and purification processes of $o$-BP single crystals. The full width at half maximum (FWHM) of X-ray rocking curve of the as-grown $o$-BP was 21.65 arc sec, indicating its respectable crystalline quality. A bottom electrode structure field-effect transistor (FET) based on the multilayer phosphorene mechanically exfoliated from the as-grown $o$-BP single crystal was successfully fabricated through an all-dry transfer technique. Impressively, the FET based on a $6 \mathrm{~nm}$ thick multilayer (approximate 12 layers) phosphorene exhibited a record high hole mobility $\left(\mu_{\mathrm{p}}\right)$ of $1744 \mathrm{~cm}^{2} \mathrm{~V}^{-1} \mathrm{~s}^{-1}$ and an admirable on/off current switching ratio $\left(I_{\text {on }} / I_{\text {off }}\right)$ of $\sim 10^{4}$, which further proved the high-quality of the o-BP single crystals synthesized by the twostep heating CVT reaction method using the simple $\mathrm{Sn} / \mathrm{I}_{2} /$ red phosphorus system.
\end{abstract}

Keywords: orthorhombic black phosphorus, single crystal, chemical vapor transport, two-step heating, field-effect transistors

\section{INTRODUCTION}

Two-dimensional (2D) layered materials, represented by graphene and transition metal dichalcogenides (TMDCs), have emerged as promising building blocks for creating functional architectures and devices due to their unique layered structures and novel physicochemical properties [1-4]. However, the zero band gap nature of graphene and the relatively low room-temperature carrier mobility in TMDCs limit their applications in nano-electronic, opto-electronic and photo-voltaic devices. Orthorhombic black phosphorus ( $o$-BP) has thus been brought back to the spotlight due to its excellent room-temperature carrier mobility and layer-dependent direct band gap, which can be tuned from 0.3 to $2 \mathrm{eV}$ as its thickness decreases from bulk to monolayer [5-8]. Therefore, $o$-BP is prized in bridging the gap between graphene and TMDCs. Accordingly, phosphorene, which is designated as a monolayer or a few layers of $o-\mathrm{BP}$, has recently emerged as a new promising member of the $2 \mathrm{D}$ material family. Based on its intriguing properties, a plethora of exciting potential applications of monolayer or multilayer phosphorene have been reported, such as field-effect transistors (FETs) [5,9-13], sodium/ lithium-ion batteries [14-18], photodetectors [19-21], gas/ion/humidity sensors [22-25], memory devices [26], and theoretically predicted for photocatalyst and thin film solar-cells $[27,28]$. Specifically, much interest has been focused on FETs based on multilayer phosphorene, stemming from its fascinating electrical properties. These FETs devices show the appreciable hole mobility $\left(\mu_{\mathrm{p}}\right)$ in the order of 10 to $10^{3} \mathrm{~cm}^{2} \mathrm{~V}^{-1} \mathrm{~s}^{-1}$ and the on/off current switching ratio $\left(I_{\text {on }} / I_{\text {off }}\right)$ of $10^{2}$ to $10^{4}[5,9-13]$. The theoretically predicted $\mu_{\mathrm{p}}$ for the monolayer or multilayer phosphorene at room temperature is infrequently high $(10,000-26,000$ $\left.\mathrm{cm}^{2} \mathrm{~V}^{-1} \mathrm{~s}^{-1}\right)[9,11]$, and the $I_{\text {on }} / I_{\text {off }}$ is remarkable large (exceeding $10^{5}$ ) $[5,9]$. The implementation of monolayer or multilayer phosphorene can be attained by several classical methods including bottom-up processes, solution-based approaches [26,29-32], top-down methods [5,9-13]. Among these, although the bottom-up direct synthesis of large-area monolayer or multilayer phosphorene is the most promising way, by its reality, it has not yet been realized. The solution-based approaches have serious negative influences on both quality and purity of monolayer or multilayer phosphorene. The top-down methods are explored to achieve monolayer or multilayer phosphorene

\footnotetext{
${ }^{1}$ Department of Chemistry, Tsinghua University, Beijing 100084, China

${ }^{2}$ Institute of Microelectronics, Tsinghua University, Beijing 100084, China

${ }^{3}$ Tsinghua National Laboratory for Information Science and Technology (TNList), Tsinghua University, Beijing 100084, China

${ }^{\dagger}$ These two authors contributed equally to this work.

*Corresponding authors (emails: yanqf@mail.tsinghu.edu.cn (Yan Q); RenTL@tsinghua.edu.cn (Ren TL))
} 
in the same way as attaining graphene from bulk graphite. Micromechanical cleavage, represented by Scotch-tape delamination, has been demonstrated as a reliable, simple yet effective method to produce ultrathin and primitive phosphorene from $o$-BP single crystals. The universal method is useful for producing small-scale monolayer or multilayer phosphorene for fundamental research purpose. From this perspective, $o$-BP single crystal is the precursor to attain high-quality monolayer or multilayer phosphorene.

The first synthesis of $o$-BP crystal can be dated back to a century ago by using super-high-pressure method [33,34]. With the development and maturation of super-high-pressure technology, more and more attentions were paid to comprehending the reaction mechanism of super-highpressure method and eventually realizing the first $o-\mathrm{BP}$ single crystal [35-40]. However, this method requires expensive and sophisticated equipment, which limits the fundamental research, applications and commercial popularization of $o$-BP single crystal. Besides this conventional preparation technique, the controllable synthesis of $o$-BP single crystal also can be attained by other ways including recrystallization from mercury [41,42] or bismuth-flux method [42-44] and mineralizer-assisted chemical vapor transport (CVT) reaction method [45-48]. Among these, the mercury or bismuth-assisted method provides a viable path for preparing $o$-BP single crystals. However, the method involves toxic chemicals and is low-throughput. The resulting $o$-BP single crystals are small in size and of limited crystallinity. The CVT reaction method has originally been regarded as a promising approach to address the purification and crystallization of the materials [49]. This method has been recently proved effective in synthesis of $o$-BP single crystals with high-quality [45-48]. However, precious metals and toxic chemicals are usually used as starting materials in the current synthesis system based on CVT method, which incurs some unwanted by-products. For instance, five unwanted by-products, such as $\mathrm{Au}_{3} \mathrm{SnP}_{7}, \mathrm{AuSn}, \mathrm{Sn}_{4} \mathrm{P}_{3}, \mathrm{Sn}_{3} \mathrm{P}_{4}$ and $\mathrm{SnI}_{4}[45,46]$, appear upon the synthesis of $o$-BP single crystals using the $\mathrm{Au} / \mathrm{Sn} / \mathrm{SnI}_{4} /$ red $\mathrm{P}$ system. In the $\mathrm{Sn} / \mathrm{SnI}_{4} /$ red $\mathrm{P}$ system [47], the synthesis procedure was improved and the precious metal was not needed. However, the tedious synthesis process of $\mathrm{SnI}_{4}$ is still required. Owing to the increasing interest of the research community on $o$-BP single crystals, the demand of this material shows ascendant trend. There is no doubt that more breakthroughs in $o$-BP-related field are still to come. In this sense, green and controllable synthesis of high-quality, large-size $o$-BP single crystals has been a top priority issue. Thus, newer, cheaper and greener synthesis strategy is highly desired to boost the application of $o$-BP single crystals in the 2D material family.

In this paper, we demonstrate the synthesis of high-quality sub-3 millimeter-sized $o$-BP single crystals by using a two-step heating CVT reaction method. Unlike previously reported low-pressure routes by Nilges's group [45-47], the $\mathrm{Sn} / \mathrm{I}_{2}$ were employed as the mineralization additives and red phosphorus was used as the precursor in the present strategy. Such a facile and green synthesis strategy could achieve a high yield of $\sim 90 \%$. Tin phosphide was the only by-product captured at the end of reaction, thus greatly simplified the subsequent separation and purification process of $o$-BP single crystals through a simple washing step with hydrochloric acid. Furthermore, it was found the emergence of the tin phosphide intermediate compound played a crucial role in the crystallization of $o-\mathrm{BP}$. The crystalline quality of the as-grown $o$-BP single crystals was proved by a series of structural and optical characterizations. Specifically, the X-ray rocking-curve data of the sub-3 millimeter-sized crystal directly indicated its respectable crystalline quality. The Tauc Plot calculation showed that the as-grown $o$-BP single crystal had a direct and narrow band gap of $0.284 \mathrm{eV}$ in its bulk form. Most importantly, the material quality of the $o$-BP single crystals was further examined by exploring the electrical properties of an FET based on multilayer phosphorene. The multilayer phosphorene was mechanically exfoliated from the as-grown $o$-BP single crystals, and then transferred onto the $5 \mathrm{~nm} \mathrm{Ti} / 25 \mathrm{~nm}$ Au bottom electrodes onto a $300 \mathrm{~nm} \mathrm{SiO} / \mathrm{p}$-Si substrate through an alldry transfer technique that did not involve any wet chemistry steps [50]. Impressively, the fabricated FET based on $6 \mathrm{~nm}$ thick phosphorene exhibited a record high $\mu_{\mathrm{p}}$ of 1744 $\mathrm{cm}^{2} \mathrm{~V}^{-1} \mathrm{~s}^{-1}$ and an excellent $I_{\text {on }} / I_{\text {off }}$ ration of $\sim 10^{4}$.

\section{EXPERIMENTAL SECTION}

\section{Crystal growth}

Sub-3 millimeter-sized $o$-BP single crystals were synthetized by the reaction of red phosphorus (500 mg, $99.999+\%$, lump, Alfa Aesar), tin (1000 mg, 99.999\%, granule, Alfa Aesar) and iodine (100 mg, 99.99+\%, crystalline, Alfa Aesar) in an evacuated silica glass ampule $(p=0.1 \mathrm{~Pa}$, length 100 $\mathrm{mm}$, inner diameter $8 \mathrm{~mm}$ and wall thickness $1 \mathrm{~mm}$ ). The ampule was horizontally placed in a tube furnace with two independent heating zones (MTI KJ OTF-1200X-III-S, Hefei, China). The two-step heating synthesis process involved a series of temperature-programmed reactions, constant temperature heating, and slow cooling treatment. Firstly, in the first-step heating reaction, the reaction starting materials were put at one side of the silica ampule and located at the high temperature zone $\left(T_{1}\right)$ of the furnace. It 
was heated from room temperature (RT) to $460^{\circ} \mathrm{C}$ in $7 \mathrm{~h}$, and then kept for $5-10 \mathrm{~h}$ at $460^{\circ} \mathrm{C}$. The empty side of the silica ampule was located at the low temperature zone $\left(T_{2}\right)$, which was simultaneously heated from RT to $400^{\circ} \mathrm{C}$ in $7 \mathrm{~h}$. Secondly, the setting temperatures were kept for 5-10 h. Subsequently, in the second-step heating reaction, the hot and low temperature zones were further heated to $630^{\circ} \mathrm{C}$ $\left(T_{1}\right)$ and $580^{\circ} \mathrm{C}\left(T_{2}\right)$ at a slow heating rate of $25^{\circ} \mathrm{C}$ per hour, respectively, and the setting temperatures were kept for 5-10 h. After that, the silica glass ampule was cooled to ambient temperature with a slow cooling rate of $50^{\circ} \mathrm{C}$ per hour. Finally, sub-3 millimeter-sized $o$-BP single crystals were obtained.

\section{Characterization of the $o$-BP single crystal}

The phase analysis of $o$-BP single crystals was carried out by using X-ray diffraction (XRD) (Bruker D8 Advance, Germany) with $\mathrm{Cu} \mathrm{Ka}$ radiation $(\lambda=1.5406 \AA)$ at $40 \mathrm{kV}$ and $40 \mathrm{~mA}$. The crystalline quality of the $o$-BP crystals was examined by using the X-ray rocking curve measurement (S2, Japan). The Raman spectra were collected via a high-resolution confocal Raman system (Horiba Jobin Yvon LabRAM HR Evolution, France) equipped with a $532 \mathrm{~nm}$ laser source. The IR transmission spectra and absorption spectra were collected via a micro-area IR system (V70/HYPERION 1000, USA). For quantitative analysis of the $o$-BP single crystal, a fraction of the as-grown crystals (approximate $50 \mathrm{mg}$ ) was dissolved in $\mathrm{HNO}_{3}$ at $120^{\circ} \mathrm{C}$ and an inductively coupled plasma-optical emission spectrometry (ICP-OES) measurement was performed (iCAP6300 ThermoFisher, USA). The chemical compositions of the crystals were identified by using a scanning electron microscope (SEM) (Hitachi SU8010, Japan) equipped with an energy dispersive X-ray spectroscope (EDX). The valences of $\mathrm{P}$ element were analyzed by using X-ray photoelectron spectra (XPS) (Escalab 250Xi, UK). The thermogravimetry and differential thermogravimetry (TG-DTG) analysis were carried out by using simultaneous thermal analyzer (STA 449F3, Germany). The thickness and surface morphology of the multilayer phosphorene were characterized through atomic force microscopy (AFM). The detailed nanostructures and chemical compositions of multilayer phosphorene were examined by a field emission transmission electron microscope (FETEM, JEOL JEM 2100F, Japan) equipped with an EDX. The cross-sectional TEM specimens were prepared by using liquid-phase exfoliation technique [29]. The dispersed nanoflakes were transferred onto a copper-carbon TEM grid. The obtained samples were then lamped for 20 min to dry out the solvent and immediately loaded into the microscope for TEM charac- terization. Optical images were taken by a microscope with a CCD camera (Olympus MX51, China) in ambient atmosphere.

\section{Fabrication and device characterization of FET}

Highly doped p-type silicon substrates capped with $300 \mathrm{~nm}$ $\mathrm{SiO}_{2}$ were used to fabricate a multilayer phosphorene FET. A patterned $5 \mathrm{~nm}$ thick Ti layer and $25 \mathrm{~nm}$ thick Au layer as bottom electrodes were deposited on the surface of $\mathrm{SiO}_{2}$ by using a sputter system (Kurt J. Lesker PVD75, USA). The FET tests were performed by using a probe station test system (Aglient B1500, USA) equipped with micromanipulator, optical microscope and white light source. It is important to note that the tests were conducted in a relatively dark room and ambient condition to prevent light and temperature induced variation of charge transport.

\section{RESULTS AND DISCUSSION}

\section{Structure characterization of the $o$-BP single crystal}

Two-step heating synthesis of high-quality o-BP single crystals in an evacuated silica ampule by using CVT reaction method is closely dependent on a temperature gradient. In order to establish the temperature gradient in a controlled manner, a tube furnace with two independent heating zones was employed, as schematically illustrated in Fig. 1a. The solid-gas reaction in the silica ampule can be divided into three regions: the forward reaction region where the solid starting materials are located at the dissolution site at a specific temperature $\left(T_{1}\right.$, see the Experimental Section); the gas motion region where the gas reaction mainly takes place through molecular diffusion; the crystallization reaction region where the target $o$-BP single crystals and the unwanted by-product tin phosphide are collected at a specific temperature $\left(T_{2}\right.$, see the Experimental Section). Among the three regions, the gas motion is the reaction-rate-determining step. In order to obtain high-quality $o$-BP single crystals, an essential factor is to achieve a relative low transport rate. Therefore, the silica ampule was horizontally placed to keep convection as part of the gas motion as small as possible. Another crucial factor is the driving force of molecular diffusion, i.e., the partial pressure gradient, which can be accurately controlled by a temperature gradient provided by a tube furnace with two independent heating zones. Sub-3 millimeter-sized $o$-BP single crystals with metallic luster were obtained through a series of temperature-programmed reactions, constant temperature heating and slow cooling treatment, as shown in Fig. $1 b$.

Fig. 2a shows the XRD pattern of the as-grown black 
a

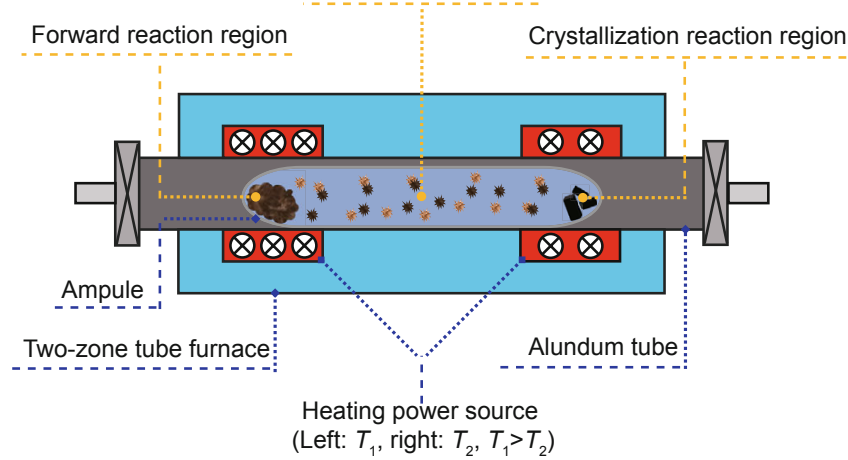

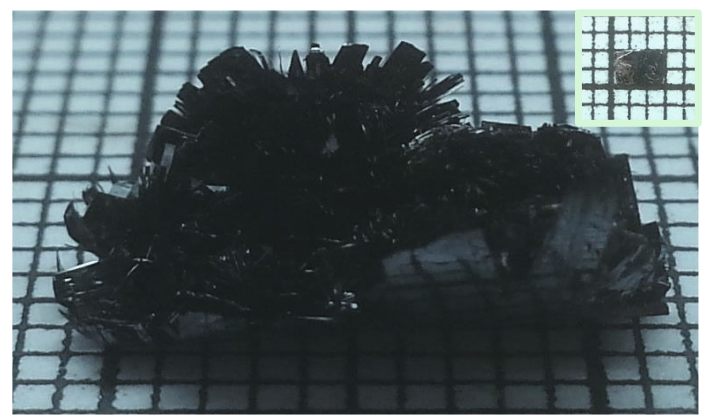

Figure 1 (a) Schematic of the experimental setup for CVT reaction in a tube furnace with two independent heating zones. (b) Photograph of the $o$-BP single crystals. Inset is the photograph of an as-grown $o$-BP single crystal of $2 \mathrm{~mm} \times 3 \mathrm{~mm}$ in size.

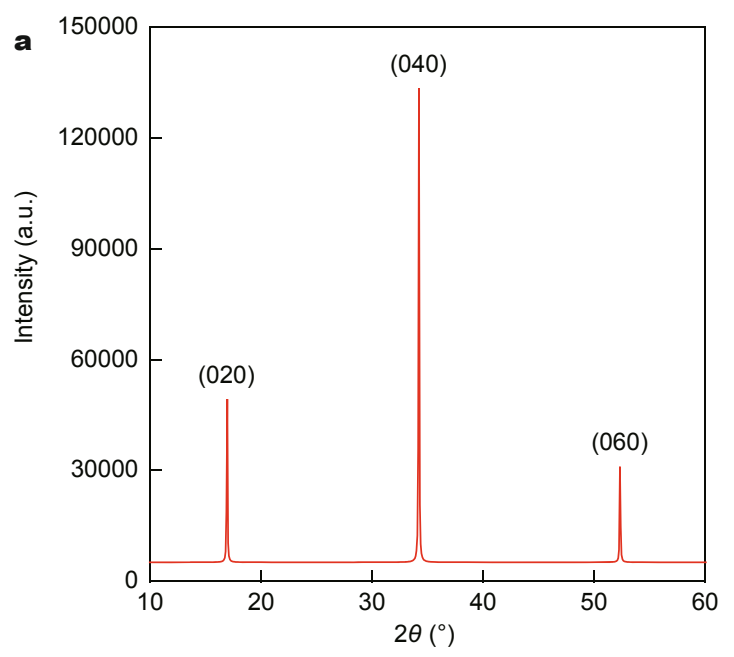

c

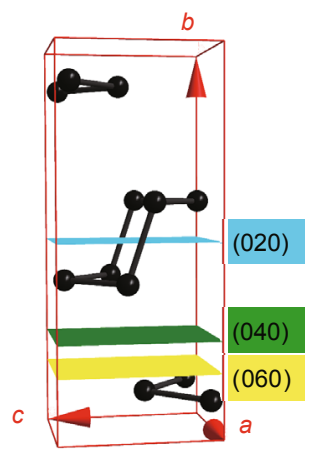

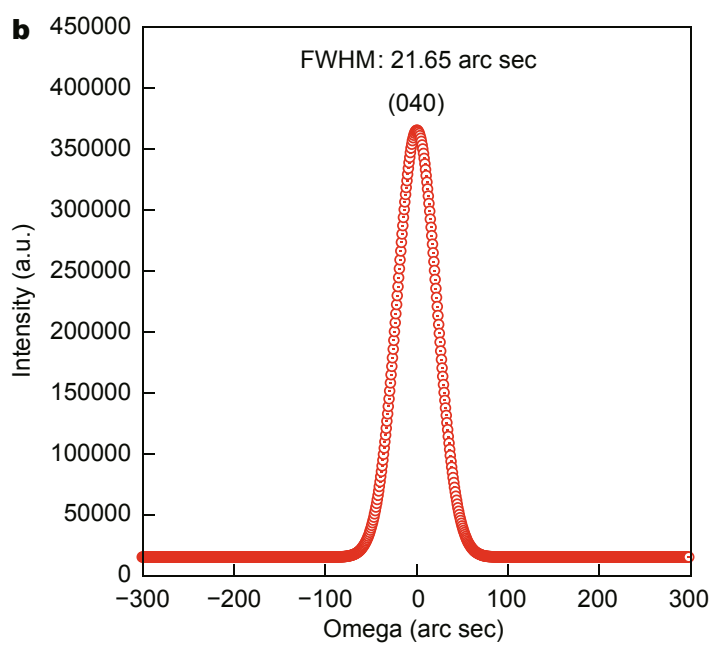

d

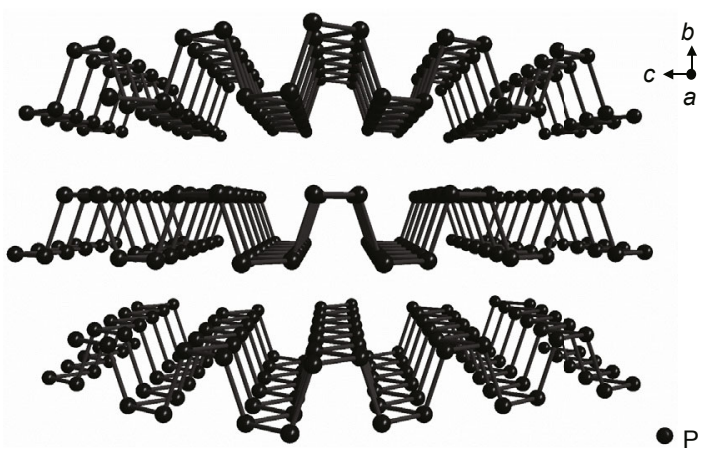

Figure 2 (a) XRD of the as-grown $o$-BP single crystal. (b) The X-ray rocking curve of the $o$-BP single crystal. (c) Schematic illustration of the $o$-BP unit cell (the (020) diffraction plane (blue), the (040) diffraction plane (green) and the (060) diffraction plane (yellow)). (d) Schematic diagram of the stacking structure of the $o$-BP single crystal.

phosphorus crystals. Careful examination of all the crystals confirms that there is no crystalline residue other than black phosphorus, and these crystals are not only pure in composition, but also in high crystalline phase. As shown in Fig. 2a, only observable characteristic diffraction peaks from the (020), (040) and (060) planes can be seen from the XRD pattern of the black phosphorus crystals, revealing that these crystals belong to the orthorhombic system (space group Cmce (No. 64), $a=3.316(1) \AA, b=10.484(2)$ $\AA, c=4.379(1) \AA)$ at RT, similar to that reported in the 
literature [45,51]. Furthermore, the intensity of these diffraction peaks is extremely high, demonstrating its single-crystalline nature. As an important index to evaluate the crystalline quality of single crystal, the full width at half maximum (FWHM) of X-ray rocking curve has been widely employed [52]. As shown in Fig. 2b, the high-resolution X-ray rocking curve measurement of the $o$-BP crystals shows a strong peak, corresponding to the (040) diffraction peak in the $2 \theta$ scan mode. The FWHM of the peak is 21.65 arc sec, indicating that the as-grown black phosphorus is a single crystal with respectable crystalline quality. To the best of our knowledge, it is the first report of the X-ray rocking curve data of $o$-BP single crystal. In $o$-BP, each $\mathrm{P}$ atom has a $3 \mathrm{~s}^{2} 3 \mathrm{p}^{3}$ valence shell configuration, therefore there are five valence shell electrons available for bonding. Fig. $2 c$ shows the unit cell consisting of puckered layers parallel to the $a c$ plane. In a single puckered layer, each $\mathrm{P}$ atom bonds to three neighboring $\mathrm{P}$ atoms through $\mathrm{sp}^{3}$ hybridized orbitals. The $\mathrm{P}$ atoms are strongly bonded in-plane, thereby forming a honeycomb network layer. Thus, each $\mathrm{P}$ atom also has left a lone pair of electrons, which makes the layers weakly interact through van der Waals forces, as schematically shown in Fig. 2d.

The above results prove the chemical reaction kinetics and thermodynamics feasibility for two-step heating synthesis of $o$-BP single crystals by using CVT reaction method in the $\mathrm{Sn} / \mathrm{I}_{2} /$ red $\mathrm{P}$ system. In the first-step heating reaction ( $T_{1}$ from RT to $460^{\circ} \mathrm{C}$ and $T_{2}$ from RT to $400^{\circ} \mathrm{C}$ ), only the tin phosphide $\left(\mathrm{Sn}_{4} \mathrm{P}_{3}\right)$ and tin phosphide iodide $\left(\mathrm{Sn}_{24} \mathrm{P}_{19.3} \mathrm{I}_{8}\right)$ compounds were collected as the final products, as shown in Fig. 3. In the second-step heating reac-

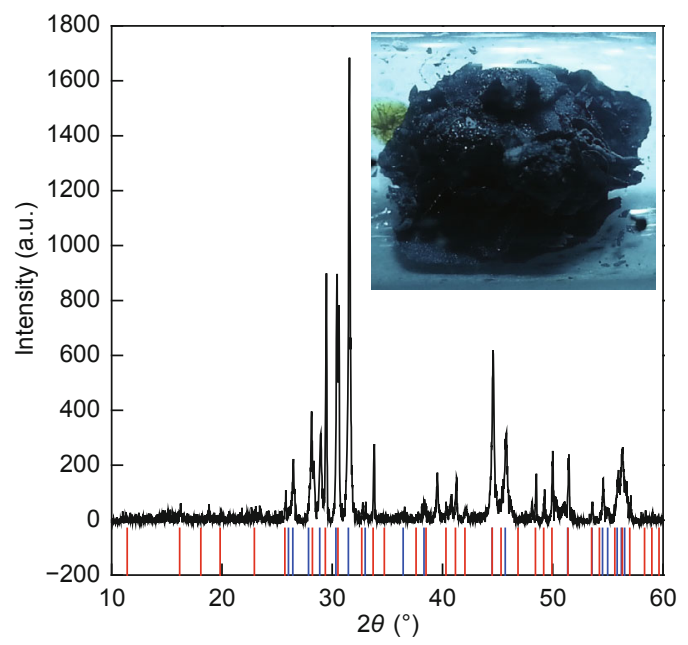

Figure 3 XRD of the compounds $\mathrm{Sn}_{4} \mathrm{P}_{3}$ (blue line, PDF\#20-1294) and $\mathrm{Sn}_{24} \mathrm{P}_{19.3} \mathrm{I}_{8}$ (red line, PDF\#52-0212) obtained at the end of first-step heating stage. Inset is the photograph of the obtained tin phosphide and tin phosphide iodide compounds. tion ( $T_{1}$ from 460 to $630^{\circ} \mathrm{C}$ and $T_{2}$ from 400 to $580^{\circ} \mathrm{C}$ ), the high-quality $o$-BP single crystals were crystallized from the tin phosphide and tin phosphide iodide compounds. It was found that no significant amounts of any other element than $\mathrm{P}$ (such as tin, iodine, or silicon and oxygen from the silica ampule material) appeared in the final product. However, a small quantity of tin phosphide crystalline residue was also detected in the cold section of the silica ampule. Although the exact chemical reaction mechanism is still not explicit in this system, we hold the opinion that both the tin phosphide intermediate compound obtained at the first-step heating stage and iodine play a decisive role in the two-step synthesis of $o$-BP single crystal. It should be stated that the $\mathrm{Sn} / \mathrm{I}_{2} /$ red $\mathrm{P}$ system has an inherent difference from the $\mathrm{Sn} / \mathrm{SnI}_{4} /$ red P system that was reported by Nilges's group [47]. In their study, an excess of tin was used to allow a quantitative transformation of $\mathrm{SnI}_{4}$ to $\mathrm{SnI}_{2}$ and to avoid $\mathrm{SnI}_{4}$ decomposition and thus formation of $\mathrm{I}_{2}$. Thus, it was believed that the $\mathrm{I}_{2}$ was unnecessary and harmful in their system [47]. Furthermore, in contrast to the $\mathrm{Sn} / \mathrm{SnI}_{4} /$ red $\mathrm{P}$ system, the present strategy is more facile and green, owing to avoiding the use of expensive and toxic $\mathrm{SnI}_{4}$.

\section{Raman spectroscopy and IR spectroscopy of the o-BP single crystal}

As an integral part of the 2D materials family research, $\mathrm{Ra}-$ man spectroscopy has been widely employed to examine the quality of the 2D materials [53]. In addition, Raman spectroscopy provides insight into all $\mathrm{sp}^{3}$-bonded $\mathrm{P}$ allotropes. Fig. 4a shows the collected Raman spectroscopy of the as-grown $o$-BP single crystal. It presents three prominent peaks at 361,436 and $463 \mathrm{~cm}^{-1}$, which are attributed to the vibrations of the $\mathrm{A}_{\mathrm{g}}^{1}, \mathrm{~B}_{2 \mathrm{~g}}^{1}$ and $\mathrm{A}_{\mathrm{g}}^{2}$ phonon mode [54-56], as shown in Fig. $4 \mathrm{~b}$. In these $\mathrm{B}_{2 \mathrm{~g}}$ and $\mathrm{A}_{\mathrm{g}}^{2}$ modes the $\mathrm{P}$ atoms oscillate within the layer plane while in the $\mathrm{A}_{\mathrm{g}}^{1}$ phonon mode the $\mathrm{P}$ atoms vibrate out-of-plane. The Raman characterization result again shows that the black phosphorus is of orthorhombic system. The optical properties of the asgrown $o$-BP single crystal were also investigated by IR spectroscopy. Fig. 4c shows the IR transmission spectroscopy for three sub-3 millimeter-sized o-BP single crystals with different thickness (red line: $60 \mu \mathrm{m}$, blue line: $80 \mu \mathrm{m}$ and black line: $100 \mu \mathrm{m})$. The dramatic change in transmittance can be attributed to the different thickness. Nevertheless, these $o$-BP single crystal samples are transparent in the IR spectrum, especially showing strong transmittance in the middle IR region. Fig. $4 \mathrm{~d}$ shows the IR absorption spectra of the $60 \mu \mathrm{m}$ thick $o$-BP single crystal (red line). The spectra show clear band edge cutoffs with no absorption tail, indicating high-quality single crystal with low defect con- 


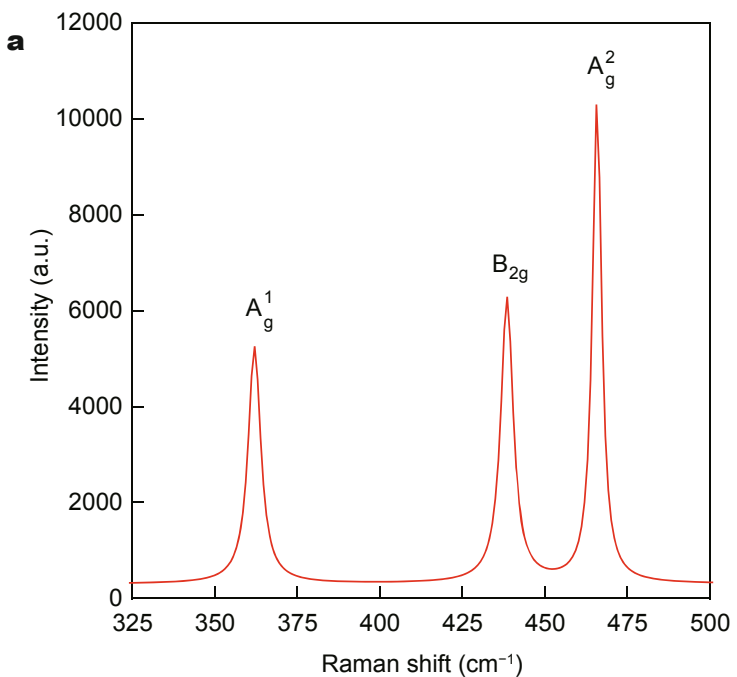

b<smiles>[AlH2]</smiles><smiles></smiles>

$\mathrm{B}_{2 \mathrm{~g}}$

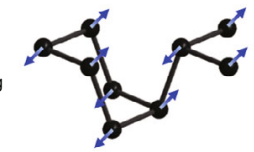

$\mathrm{A}_{9}^{2}$
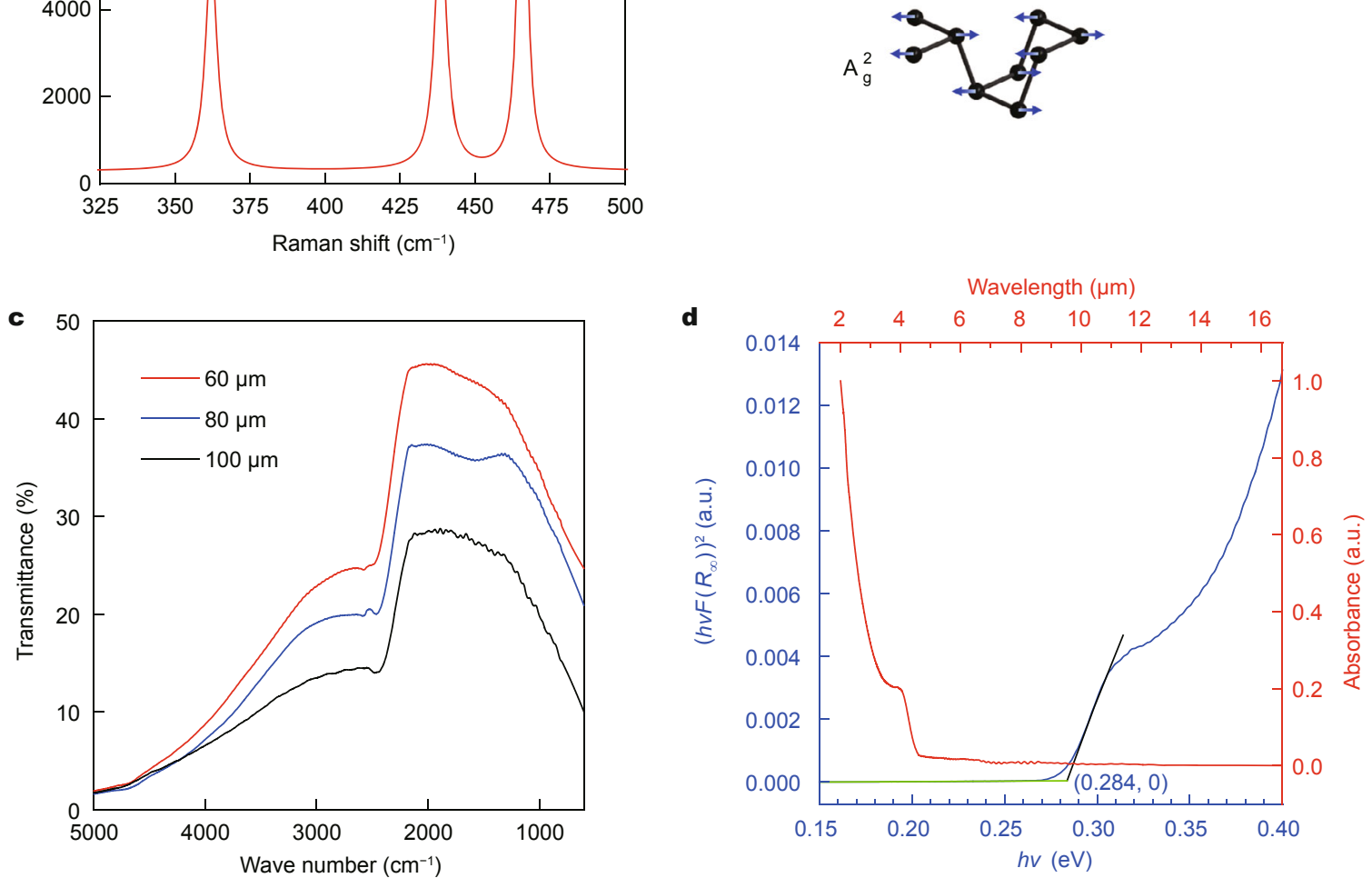

Figure 4 (a) Typical Raman spectra of the as-grown $o$-BP single crystal. (b) Three Raman-active vibrational modes of the $o$-BP lattice. (c) IR transmission spectra for three sub-3 millimeter-sized $o$-BP single crystals with different thickness (red line: $60 \mu \mathrm{m}$, blue line: $80 \mu \mathrm{m}$ and black line: $100 \mu \mathrm{m}$ ). (d) Plots of IR absorption of the $60 \mu \mathrm{m}$ thick $o$-BP single crystal (red line), $h v-\left(h v F\left(R_{\infty}\right)\right)^{2}$ curve of this sample (blue line) and two tangent line of the $h v$ $\left(h v F\left(R_{\infty}\right)\right)^{2}$ curve (green line: $Y_{1}=0$ and black line: $\left.Y_{2}=0.155 X_{2}-0.044\right)$.

centration. The band gap value of $o$-BP single crystal was calculated based on the Tauc Plot method [57,58], following relational expression: $\left(h v F\left(R_{\infty}\right)\right)^{1 / n}=A\left(h v-E_{\mathrm{g}}\right)$, where $h$ is the Planck's constant, $v$ is the frequency of vibration, $E_{g}$ is the band gap, $A$ is the proportional constant. The value of the exponent $n$ denotes the nature of the sample transition. For direct allowed transition $n=1 / 2$, direct forbidden transition $n=3 / 2$, indirect allowed transition $n=2$ and indirect forbidden transition $n=3$. As $o$-BP single crystal is a direct band gap elemental semiconductor, $n$ value is $1 / 2$ [5,59]. Fig. $4 \mathrm{~d}$ shows the $h v-\left(h v F\left(R_{\infty}\right)\right)^{2}$ curve of $60 \mu \mathrm{m}$ thick $o$-BP single crystal (blue line) and two tangent line of the $h v-\left(h v F\left(R_{\infty}\right)\right)^{2}$ curve (green line: $Y_{1}=0$ and black line:
$\left.Y_{2}=0.155 X_{2}-0.044\right)$. By extrapolating the linear region of the $Y_{2}$ to the $h v$-axis intercept, as shown in Fig. $4 \mathrm{~d}, E_{\mathrm{g}}$ is established. It is determined that the $o$-BP single crystal has a band gap of $0.284 \mathrm{eV}$, which is generally consistent with prior measurements $[5,59]$.

\section{ICP-OES, EDX and XPS characterizations of the $o$-BP single crystal}

ICP-OES characterization was performed on the as-grown $o$-BP single crystal, indicating a purity of 99.0 at.\%. It was found that no significant amounts of any other element than $\mathrm{P}$ appeared in the final product. EDX characterization of the as-grown $o$-BP single crystal is illustrated in Figs $5 \mathrm{a}$, 
b and c. Fig. 5a shows that the chemical composition of the $o$-BP single crystal is pure $\mathrm{P}$ without other elements (such as tin, iodine, or silicon and oxygen from the silica ampule material). To clarify this point further, EDX element mapping characterization was employed by selecting a surface area of the crystal at random, as shown in Figs 5b and c. The result demonstrates the $\mathrm{P}$ element is homogeneously distributed in the sample and rules out the possibility of emergence of other elements. The valences of $\mathrm{P}$ element were analyzed by using XPS, as shown in Fig. $5 \mathrm{~d}$. The asgrown $o$-BP single crystal only has the $2 \mathrm{p}^{3 / 2}$ and $2 \mathrm{p}^{1 / 2}$ doublet at 130.5 and $131.3 \mathrm{eV}$, respectively, which is the characteristic of $o$-BP [30]. Other sub-bands of P element are not apparent. Therefore, the possibility of forming any $\mathrm{P}$ element based compound can be eliminated out. The above results clear and convincing evidences again prove that the synthesized single crystal is indeed orthorhombic black phosphorus.

\section{TG-DTG characterization of the $o$-BP single crystal}

To examine the thermal stability of the $o$-BP single crystals, TG-DTG analysis were carried out under different ambient atmospheres (argon flow and air flow) from RT to $900^{\circ} \mathrm{C}$. Fig. 6a shows the TG curve and DTG curve of $o$-BP single crystal under argon flow. The sample undergoes weight loss in a single step, with the onset temperature at $330^{\circ} \mathrm{C}$, suggesting a clear endothermic decomposition process. In a word, the pure $o$-BP single crystal decomposes in a single mass-loss step at a well-defined temperature under argon flow. Such a decomposition process can be attributed to
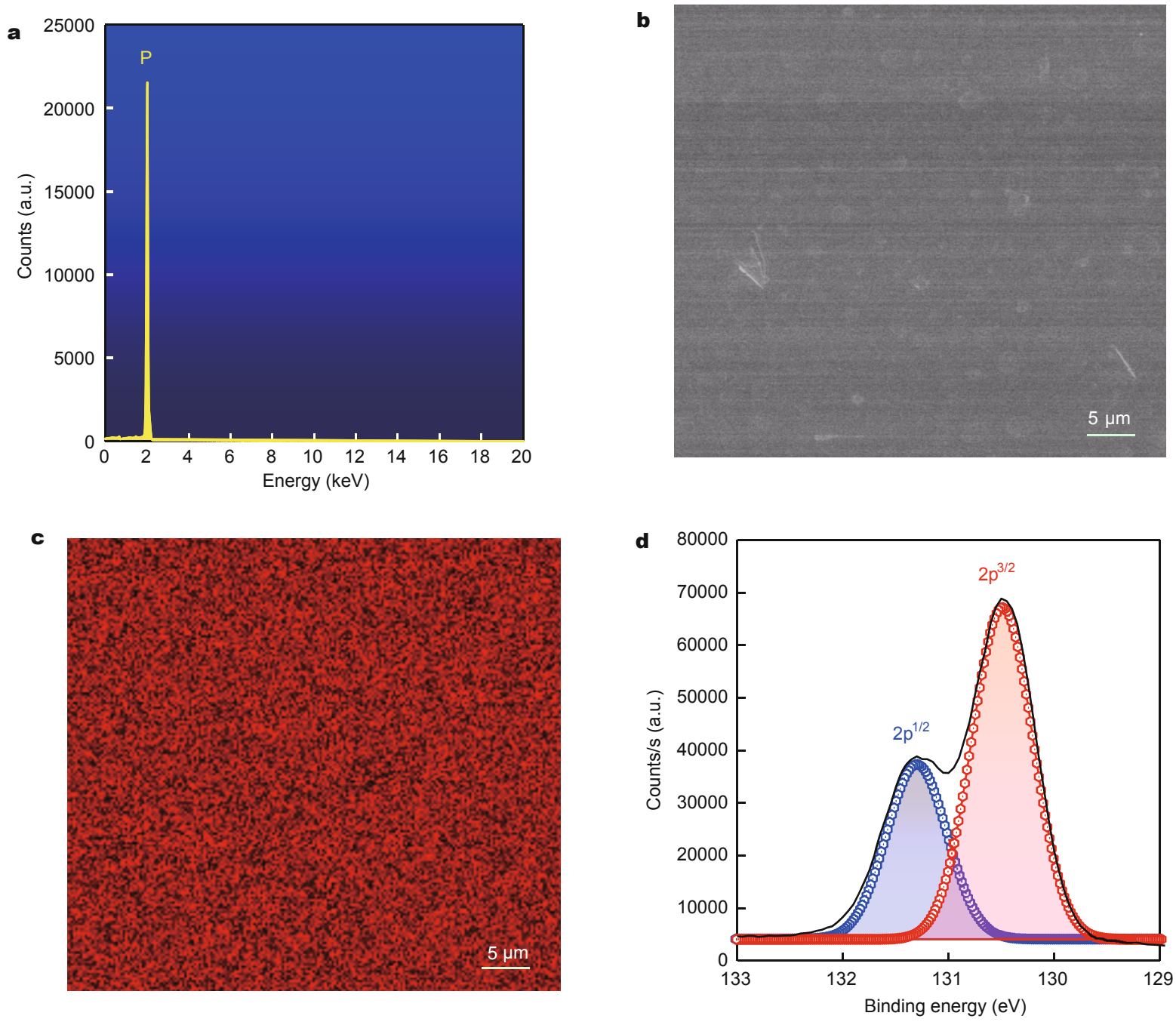

Figure 5 (a) EDX analysis of the as-grown $o$-BP single crystal. (b) SEM image of the as-grown $o$-BP single crystal. (c) EDX mapping of P element, corresponding to the SEM image in (b). (d) XPS characterization of the as-grown $o$-BP single crystal. 

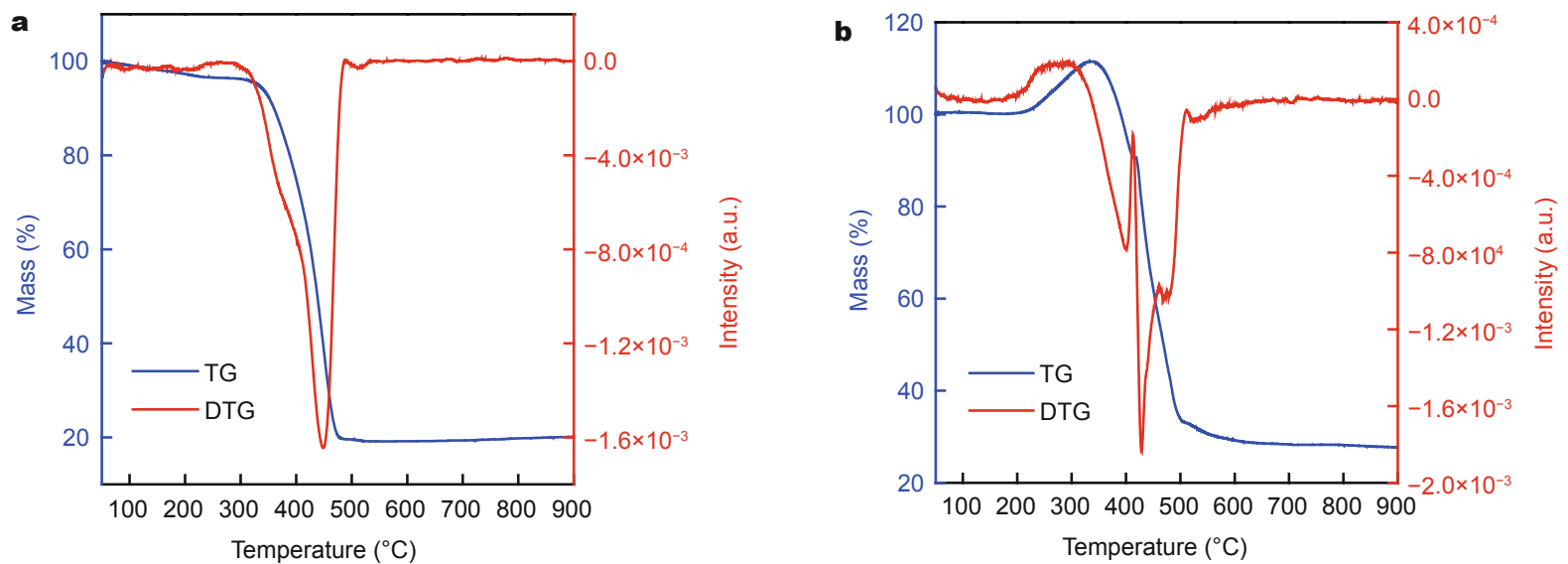

Figure 6 The thermal stability of the $o$-BP single crystals. (a) The TG-DTG curves of $o$-BP single crystal under argon flow. (b) The TG-DTG curves of $o$-BP single crystal under air flow.

gasification $\left(\mathrm{P}_{(\mathrm{s})} \rightarrow \mathrm{P}_{(\mathrm{g})}\right)$ caused by the high temperature effect. However, a different thermal behavior was observed in $o$-BP single crystal under air flow as both TG and DTG curves showed three thermal events occurring in temperature range from RT to $900^{\circ} \mathrm{C}$, as shown in Fig. 6b. The $o$-BP crystal first undergoes $10 \%$ mass gain at $200^{\circ} \mathrm{C}$, which is an exothermic process and can be attributed to the oxidation of a portion of $o$ - $\mathrm{BP}$ crystal $\left(\mathrm{P}_{(\mathrm{s})} \rightarrow \mathrm{P}_{x} \mathrm{O}_{y(\mathrm{~s})}\right)$ without the help of water. It is speculated that oxygen existing in the air would insert into the lattice of $o$-BP crystal, which has overcome an activation barrier upon heating [60]. Oxygen insertion results in considerable deformation of $o$-BP crystal. This promotes further oxidation of BP in the neighborhood of the interstitial oxygen defects. For high enough interstitial oxygen concentration, a portion of crystal might form $\mathrm{P}_{x^{-}}$ $\mathrm{O}_{y(\mathrm{~s})}$ compound. Subsequently, this sample begins to undergo $20 \%$ weight loss at $330^{\circ} \mathrm{C}$ with a clear endothermic decomposition process. The decomposition temperature is consistent with the thermal behavior under argon flow, indicating that the unoxidized $o$-BP single crystal begins to decompose $\left(\mathrm{P}_{(\mathrm{s})} \rightarrow \mathrm{P}_{(\mathrm{g})}\right)$. Finally, this sample begins to suffer severe weight loss at $410^{\circ} \mathrm{C}$ with a similar endothermic decomposition process. Comparing these two samples, it can be deduced that the $o$-BP single crystals appear to be more stable under the inert gases (e.g., argon), for no sign of thermal decomposition is observed until $330^{\circ} \mathrm{C}$.

\section{TEM characterization of multilayer phosphorene} exfoliated from the as-grown o-BP single crystal

Phosphorene is the building block for creating nano-electronics devices. In order to ensure the high crystalline quality of monolayer or multilayer phosphorene exfoliated from the as-grown $o$-BP single crystal, their detailed nano- structures were characterized by TEM and EDX element mapping, as shown in Fig. 7. Phosphorene was produced by employing the liquid-phase exfoliation technique [29]. Fig. 7a shows a typical TEM image of a multilayer phosphorene on copper-carbon grid. To clarify that the sample was not polluted during preparation, EDX element mapping was used to collect the chemical compositions of the selected area (outlined in white dotted line), as shown in the inset of Fig. 7a. The EDX element mapping result demonstrates that the sample only consists of $\mathrm{P}$ element, without any impurity components. The high-resolution TEM imaging was employed to study the atomic structure of the phosphorene with the $(0 \mathrm{k} 0)$ crystal plane, as shown in Fig. 7b. The outlined in red dotted line of the schematic diagram of $(0 \mathrm{k} 0)$ crystal plane in lower right in Fig. $7 \mathrm{~b}$ has a good match with the $d=2.82 \AA$ measured in TEM image. The orthorhombic system characteristic of the black phosphorus single crystal was also confirmed by selected area electron diffraction (SAED) (upper left inset of Fig. $7 b)$. These lattice parameters perfectly match the results reported in literature [29].

\section{FET based on multilayer phosphorene exfoliated from the as-grown o-BP single crystal}

To further examine the material quality of the as-grown $o$-BP single crystals, device performance of an FET fabricated through a "photolithography-free" method based on multilayer phosphorene was explored [50]. The multilayer phosphorene was first exfoliated onto the poly-vinyl chloride (PVC) film using a mechanical exfoliation method. Secondly, the target sample was placed above a certain electrode patterned substrate $(25 \mathrm{~nm} \mathrm{Au} / 5 \mathrm{~nm}$ Ti bottom electrodes onto a $300 \mathrm{~nm} \mathrm{SiO} / \mathrm{p}$-Si substrate), with the help 
a

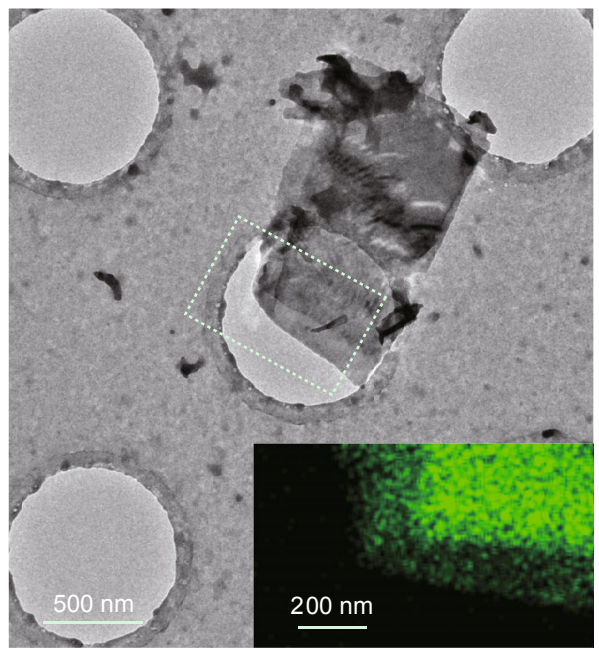

b

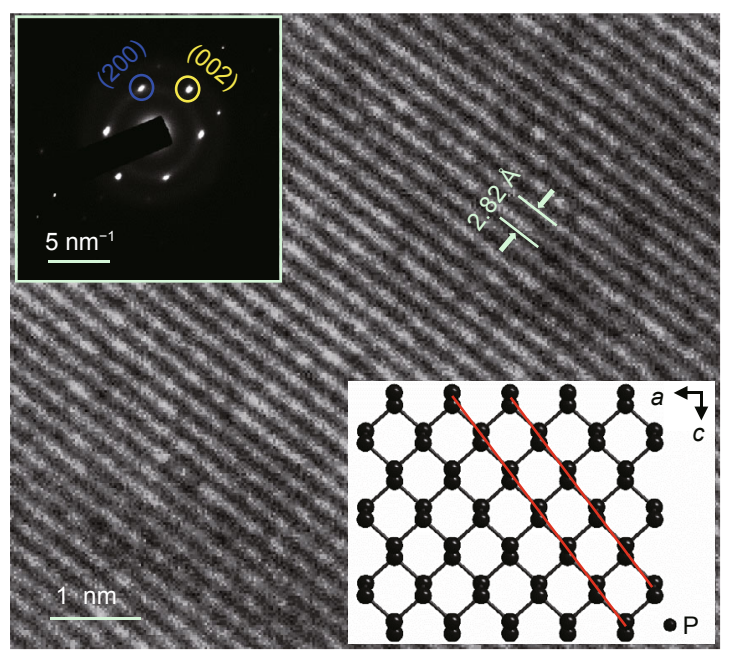

Figure 7 (a) Typical TEM image of a multilayer phosphorene on copper-carbon support with P element EDX mapping (green, lower right inset) of the selected area (outlined in green dotted line). (b) High-resolution TEM image of phosphorene taken from the selected area (outlined in green dotted line in (a)) with the schematic atomic structure of (0k0) crystal plane (lower right inset), as well as SAED pattern of the selected area (upper left inset).

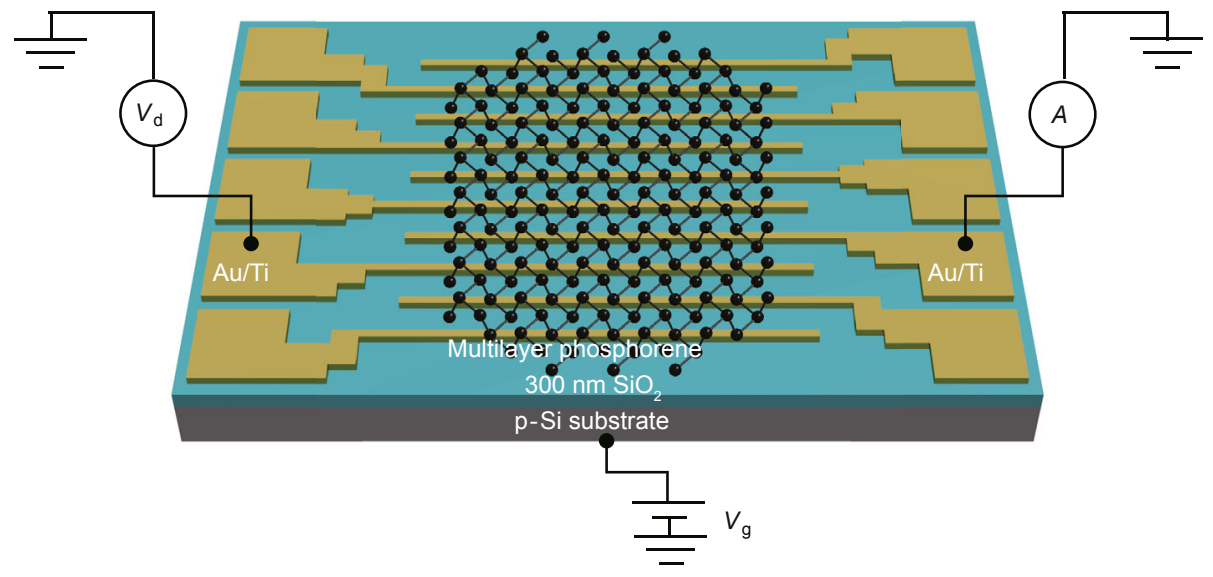

Figure 8 Schematic diagram of an FET based on multilayer phosphorene.

of a micro-manipulation platform. Subsequently, upon slowly moving down the PVC film, the film would closely attach the electrode patterned substrate due to the PVC film's self-adsorption characteristic. Finally, after lifting up the film, the target sample would stay on the electrode with high probability. In order to improve the contact between the target sample and electrode patterned substrate, an annealing process was operated. The sample was heated in nitrogen at $200^{\circ} \mathrm{C}$ for $20 \mathrm{~min}$. In a word, the method was an all-dry transfer technique that did not involve any wet chemistry steps. However, it should be noted that when performing annealing and transfer curve tests, the sample was indeed exposed to air in some extent, approximate
0.5-1 h. Schematic diagram of an FET based on multilayer phosphorene is shown in Fig. 8.

The obtained multilayer phosphorene was attached to the bottom electrodes consisting of $5 \mathrm{~nm}$ Ti and 25 $\mathrm{nm} \mathrm{Au}$ onto a $300 \mathrm{~nm} \mathrm{SiO} / \mathrm{p}$-Si substrate, as shown in Fig. 9a. It should be noted that the subsequent multilayer phosphorene related characterizations were based on the sample locating at the area between the A electrode and B electrode, as illustrated in Fig. 9a. Fig. 9b shows the Raman spectra of the target sample. The prominent peaks at 362, 439 and $467 \mathrm{~cm}^{-1}$ are due to the vibrations of the $A_{\mathrm{g}}^{1}, \mathrm{~B}_{2 \mathrm{~g}}$ and $\mathrm{A}_{\mathrm{g}}^{2}$ phonon mode of multilayer phosphorene [56]. The peak at $520.6 \mathrm{~cm}^{-1}$ corresponds to the Raman-active signal 
of the silicon substrate. These results confirm that the exfoliated multilayer phosphorene is of orthorhombic phase with high-quality. AFM image was used to determine the thickness of multilayer phosphorene, the channel length and the channel width, as detailed in Fig. 9c. The values are about $6 \mathrm{~nm}, 4.4 \mu \mathrm{m}$ and $9.4 \mu \mathrm{m}$, respectively. It also shows that there had mild oxidation on the sample surface after FET device characterization. Some small white bubbles can be seen which is the indication of water uptake. The FET based on multilayer phosphorene displays excellent charge transport characteristics, as shown in Fig. 9d. We extracted the hole mobility from the slope in the linear region of the measured transfer characteristics using Equation (1),

$$
\mu=\frac{\mathrm{d} I_{\mathrm{ds}}}{\mathrm{d} V_{\mathrm{gs}}} \times \frac{L}{W C_{\mathrm{i}} V_{\mathrm{ds}}},
$$
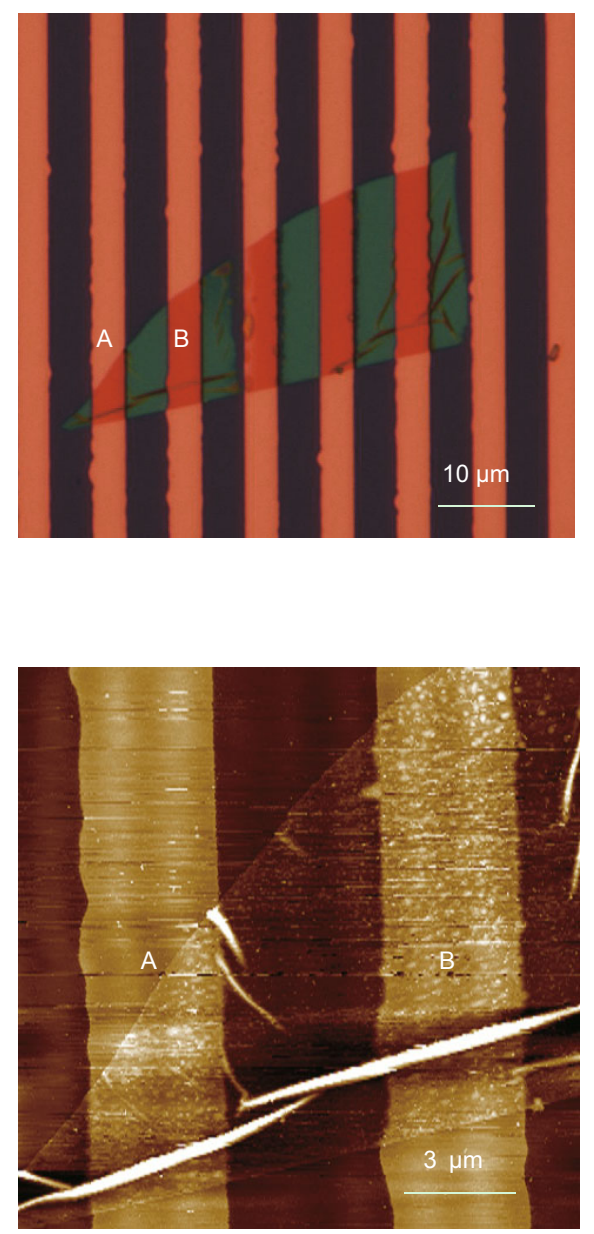

where $L$ is the length of the channel $(L=4.4 \mu \mathrm{m}), W$ is the width of the channel $(W=9.4 \mu \mathrm{m}), V_{\mathrm{ds}}$ is the source-drain bias $\left(V_{\mathrm{ds}}=0.01 \mathrm{~V}\right)$, and $C_{\mathrm{i}}$ is the capacitance between the channel and the back-gate per unit area $\left(C_{\mathrm{i}}=\varepsilon_{\mathrm{o}} \varepsilon_{\mathrm{r}} / d, \varepsilon_{\mathrm{r}}=3.9\right.$ for $\mathrm{SiO}_{2}, \varepsilon_{0}=8.85 \times 10^{-12} \mathrm{~F} \mathrm{~m}^{-1}$ for the permittivity of vacuum, $d=$ oxide thickness of $300 \mathrm{~nm}$ ). It exhibits an on-state current exceeding $1 \mu \mathrm{A}$, a record high $\mu_{\mathrm{p}}$ of $1744 \mathrm{~cm}^{2} \mathrm{~V}^{-1} \mathrm{~s}^{-1}$ and an excellent $I_{\text {on }} / I_{\text {off }}$ of $\sim 10^{4}$ when the source-drain bias is $0.01 \mathrm{~V}$. The remarkable performance of this FET device can be ascribed to the high-quality of the exfoliated multilayer phosphorene, which inherits the high crystallinity of the as-grown $o$-BP single crystal. What's more, the bottom electrode structure also provides an efficient way to construct high-performance FETs based on multilayer phosphorene because it minimizes the fabrication time and offers the opportunity to test the sample in fresh state.
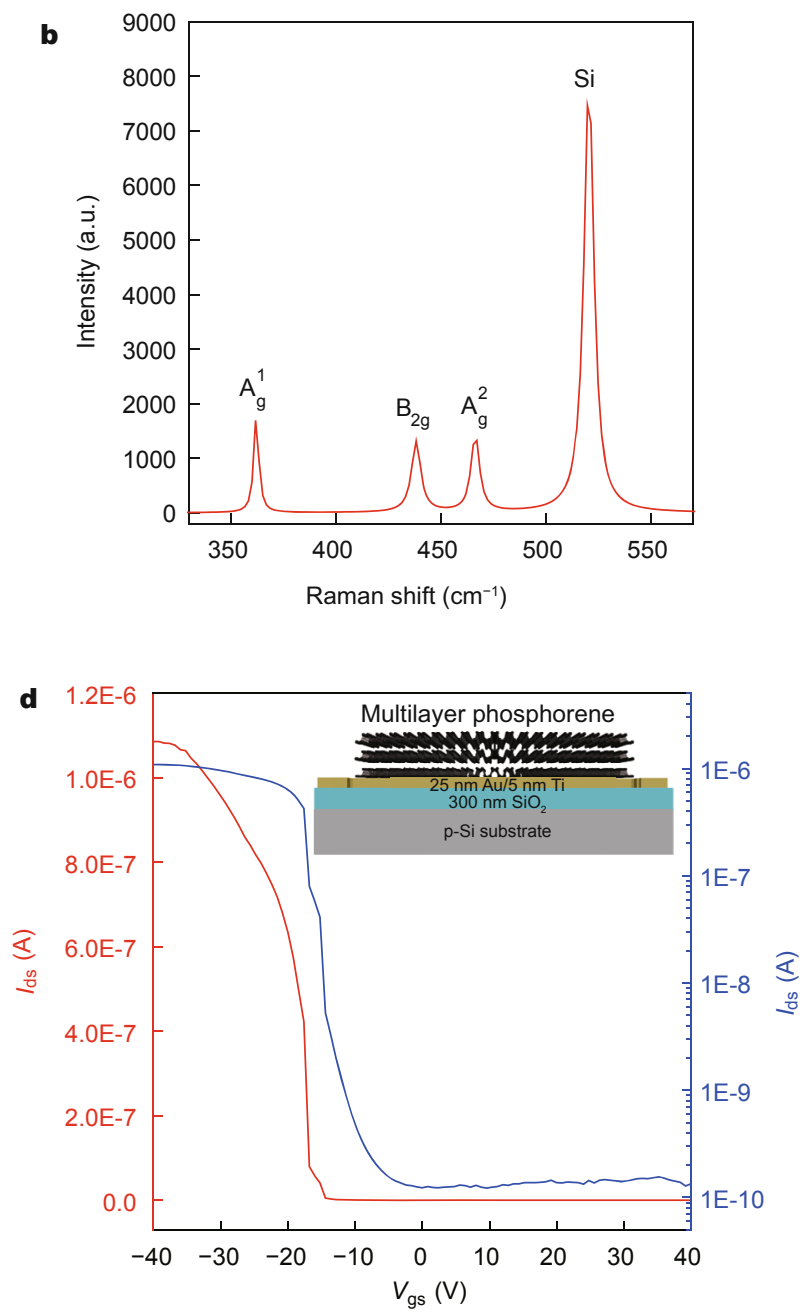

Figure 9 (a) An optical microscopy image of multilayer phosphorene with bottom electrode $(\mathrm{Au} / \mathrm{Ti} 25 \mathrm{~nm} / 5 \mathrm{~nm})$ onto a $300 \mathrm{~nm} \mathrm{SiO} / \mathrm{p}-\mathrm{Si}$ substrate. (b) Raman spectra of the target sample. (c) An AFM image of the target sample. (d) Transfer curve for a 6 nm thick multilayer phosphorene FET. The drain current $v s$. gate voltage on a linear scale (red, left) and a logarithmic scale (blue, right) with a front view of FET device schematic (upper right inset). 


\section{CONCLUSION}

In summary, a two-step heating CVT reaction method for the synthesis of high-quality sub-3 millimeter-sized $o$-BP single crystal is reported. The newer, cheaper and greener synthesis system is composed of $\mathrm{Sn} / \mathrm{I}_{2}$ as mineralization additives and red phosphorus as precursor. Such a facile synthesis strategy can achieve a high yield of $\sim 90 \%$. Tin phosphide is the only by-product captured at the end of reaction, which greatly simplifies the subsequent separation and purification processes of the $o$-BP single crystals. A series of structural and optical characterizations indicate the as-grown $o$-BP single crystals possess high crystalline quality. The Tauc Plot calculation method deduces that the as-grown $o$-BP single crystal has a direct and narrow band gap of $0.284 \mathrm{eV}$. The $o$-BP single crystals appear to be more stable under the inert gases (e.g., argon), for no signature of thermal decomposition is observed until $330^{\circ} \mathrm{C}$. Multilayer phosphorene was mechanically exfoliated from the asgrown $o$-BP single crystals. A bottom electrode structure FET based on the multilayer phosphorene was successfully fabricated through an all-dry transfer technique. Impressively, the FET based on a $6 \mathrm{~nm}$ thick multilayer phosphorene exhibited a record high $\mu_{\mathrm{p}}$ of $1744 \mathrm{~cm}^{2} \mathrm{~V}^{-1} \mathrm{~s}^{-1}$ and an admirable $I_{\text {on }} / I_{\text {off }}$ ratio of $\sim 10^{4}$, which further proved the high-quality of the $o$-BP crystals synthesized by the twostep heating CVT reaction method.

\section{Received 26 January 2016; accepted 16 February 2016;} published online 26 February 2016

1 Novoselov KS, Geim AK, Morozov SV, et al. Electric field effect in atomically thin carbon films. Science, 2004, 306: 666-669

2 Novoselov KS, Jiang D, Schedin F, et al. Two-dimensional atomic crystals. Prog Natl Acad Sci USA, 2005, 102: 10451-10453

3 Wang QH, Kalantar-Zadeh K, Kis A, et al. Electronics and optoelectronics of two-dimensional transition metal dichalcogenides. Nat Nanotechnol, 2012, 7: 699-712

4 Huang X, Zeng Z, Zhang H. Metal dichalcogenide nanosheets: preparation, properties and applications. Chem Soc Rev, 2013, 42: 1934-1946

5 Li L, Yu Y, Ye GJ, et al. Black phosphorus field-effect transistors. Nat Nanotechnol, 2014, 9: 372-377

6 Churchill $\mathrm{HOH}$, Jarillo-Herrero P. Phosphorus joins the family. Nat Nanotechnol, 2014, 9: 330-331

7 Reich ES. Phosphorene excites materials scientists. Nature, 2014, 506: 19

8 Liu H, Du Y, Deng Y, et al. Semiconducting black phosphorus: synthesis, transport properties and electronic applications. Chem Soc Rev, 2015, 44: 2732-2743

9 Xia F, Wang H, Jia Y. Rediscovering black phosphorus as an anisotropic layered material for optoelectronics and electronics. Nat Commun, 2014, 5: 4458

10 Buscema M, Groenendijk DJ, Steele GA, et al. Photovoltaic effect in few-layer black phosphorus PN junctions defined by local electrostatic gating. Nat Commun, 2014, 5: 4651

11 Qiao J, Kong X, Hu ZX, et al. High-mobility transport anisotropy and linear dichroism in few-layer black phosphorus. Nat Commun, 2014, 5: 4475

12 Miao J, Zhang S, Cai L, et al. Ultrashort channel length black phosphorus field-effect transistors. ACS Nano, 2015, 9: 9236-9243

13 Zhu W, Yogeesh MN, Yang S, et al. Flexible black phosphorus ambipolar transistors, circuits and AM demodulator. Nano Lett, 2015, 15: $1883-1890$

14 Park CM, Sohn HJ. Black phosphorus and its composite for lithium rechargeable batteries. Adv Mater, 2007, 19: 2465-2468

15 Sun LQ, Li MJ, Sun K, et al. Electrochemical activity of black phosphorus as an anode material for lithium-ion batteries. J Phys Chem C, 2012, 116: 14772-14779

16 Yao Q, Huang C, Yuan Y, et al. Theoretical prediction of phosphorene and nanoribbons as fast charging Li ion battery anode materials. J Phys Chem C, 2015, 119: 6923-6928

17 Hembram KPSS, Jung H, Yeo BC, et al. Unraveling the atomistic sodiation mechanism of black phosphorus for sodium ion batteries by first-principles calculations. J Phys Chem C, 2015, 119: 15041-15046

18 Li W, Yang Y, Zhang G, et al. Ultrafast and directional diffusion of lithium in phosphorene for high-performance lithium-ion battery. Nano Lett, 2015, 15: 1691-1697

19 Engel M, Steiner M, Avouris P. Black phosphorus photodetector for multispectral, high-resolution imaging. Nano Lett, 2014, 14: 6414-6417

20 Viti L, Hu J, Coquillat D, et al. Black phosphorus terahertz photodetectors. Adv Mater, 2015, 27: 5567-5572

21 Wu J, Koon GKW, Xiang D, et al. Colossal ultraviolet photoresponsivity of few-layer black phosphorus. ACS Nano, 2015, 9: 8070-8077

22 Kou L, Frauenheim T, Chen C. Phosphorene as a superior gas sensor: selective adsorption and distinct I-V response. J Phys Chem Lett, 2014, 5: 2675-2681

23 Abbas AN, Liu B, Chen L, et al. Black phosphorus gas sensors. ACS Nano, 2015, 9: 5618-5624

24 Li P, Zhang D, Liu J, et al. Air-stable black phosphorus devices for ion sensing. ACS Appl Mater Interfaces, 2015, 7: 24396-24402

25 Yasaei P, Behranginia A, Foroozan T, et al. Stable and selective humidity sensing using stacked black phosphorus flakes. ACS Nano, 2015, 9: 9898-9905

26 Zhang X, Xie H, Liu Z, et al. Black phosphorus quantum dots. Angew Chem Int Ed, 2015, 54: 3653-3657

27 Sa B, Li YL, Qi J, et al. Strain engineering for phosphorene: the potential application as a photocatalyst. J Phys Chem C, 2014, 118: 26560-26568

28 Dai J, Zeng XC. Bilayer phosphorene: effect of stacking order on bandgap and its potential applications in thin-film solar cells. J Phys Chem Lett, 2014, 5: 1289-1293

29 Yasaei P, Kumar B, Foroozan T, et al. High-quality black phosphorus atomic layers by liquid-phase exfoliation. Adv Mater, 2015, 27: 1887-1892

30 Kang J, Wood JD, Wells SA, et al. Solvent exfoliation of electronic-grade, two-dimensional black phosphorus. ACS Nano, 2015, 9: 3596-3604

31 Sresht V, Pádua AAH, Blankschtein D. Liquid-phase exfoliation of phosphorene: design rules from molecular dynamics simulations. ACS Nano, 2015, 9: 8255-8268

32 Woomer AH, Farnsworth TW, Hu J, et al. Phosphorene: synthesis, scale-up, and quantitative optical spectroscopy. ACS Nano, 2015, 9: $8869-8884$

33 Bridgman PW. Two new modifications of phosphorus. J Am Chem Soc, 1914, 36: 1344-1363.

34 Bridgman PW. Further note on black phosphorus. J Am Chem Soc, 1916, 38: 609-612 
35 Hultgren R, Gingrich NS, Warren BE. The atomic distribution in red and black phosphorus and the crystal structure of black phosphorus. J Chem Phys, 1935, 3: 351-355

36 Jacobs RB. Phosphorus at high temperatures and pressures. J Chem Phys, 1937, 5: 945-953.

37 Keyes RW. The electrical properties of black phosphorus. Phys Rev, 1953, 92: 580-584

38 Shirotani I, Maniwa R, Sato H, et al. Preparation, growth of large single crystals, and physicochemical properties of black phosphorus at high pressures and temperatures. Nippon Kagaku Kaishi, 1981, 10: 1604-1609

39 Shirotani I. Growth of large single crystals of black phosphorus at high pressures and temperatures, and its electrical properties. Mol Cryst Liq Cryst, 1982, 86: 203-211

40 Endo S, Akahama Y, Terada S, et al. Growth of large single crystals of black phosphorus under high pressure. Jpn J Appl Phys, 1982, 21: L482-L484

41 Krebs VH, Weitz H, Worms KH. Über die struktur und eigenschaften der halbmetalle. Viii. Die katalytische darstellung des schwarzen phosphors. Z Anorg Allg Chem, 1955, 280: 119-133

42 Maruyama Y, Suzuki S, Kobayashi K, et al. Synthesis and some properties of black phosphorus single crystals. Physica B, 1981, 105B: 99-102

43 Brown A, Rundqvist S. Refinement of the crystal structure of black phosphorus. Acta Crystallogr, 1965, 19: 684-685

44 Baba M, Izumida F, Takeda Y, et al. Preparation of black phosphorus single crystals by a completely closed bismuth-flux method and their crystal morphology. Jpn J Appl Phys, 1989, 28: 1019-1022

45 Lange S, Schmidt P, Nilges T. $\mathrm{Au}_{3} \mathrm{SnP}_{7} @$ black phosphorus: an easy access to black phosphorus. Inorg Chem, 2007, 46: 4028-4035

46 Nilges T, Kersting M, Pfeifer T. A fast low-pressure transport route to large black phosphorus single crystals. J Solid State Chem, 2008, 181: 1707-1711

47 Köpf M, Eckstein N, Pfister D, et al. Access and in situ growth of phosphorene-precursor black phosphorus. J Cryst Growth, 2014, 405: 6-10

48 Zhao M, Qian H, Niu X, et al. Growth mechanism and enhanced yield of black phosphorus microribbons. Cryst Growth Des, 2016, 16: 1096-1103

49 Schmidt P, Binnewies M, Glaum R, Schmidt M. Chemical vapor transport reactions-methods, materials, modeling. In: Ferreira SO (Ed). Advanced Topics on Crystal Growth. Rijeka: InTech, 2013: 227-306

50 Castellanos-Gomez A, Buscema M, Molenaar R, et al. Deterministic transfer of two-dimensional materials by all-dry viscoelastic stamping. 2D Mater, 2014, 1: 011002

51 Zhang CD, Lian JC, Yi W, et al. Surface structures of black phosphorus investigated with scanning tunneling microscopy. J Phys Chem C, 2009, 113: 18823-18826

52 Liu Y, Yang Z, Cui D, et al. Two-inch-sized perovskite $\mathrm{CH}_{3} \mathrm{NH}_{3} \mathrm{PbX}_{3}$ $(\mathrm{X}=\mathrm{Cl}, \mathrm{Br}, \mathrm{I})$ crystals: growth and characterization. Adv Mater, 2015, 27: 5176-5183

53 Ferrari AC, Basko DM. Raman spectroscopy as a versatile tool for studying the properties of graphene. Nat Nanotechnol, 2013, 8: 235-246

54 Sugai S, Shirotani I. Raman and infrared reflection spectroscopy in black phosphorus. Solid State Commun, 1985, 53: 753-755

55 Fei R, Yang L. Lattice vibrational modes and Raman scattering spectra of strained phosphorene. Appl Phys Lett, 2014, 105: 083120

56 Castellanos-Gomez A, Vicarelli L, Prada E, et al. Isolation and characterization of few-layer black phosphorus. 2D Mater, 2014, 1: 025001

57 Tauc J, Grigorovici R, Vancu A. Optical properties and electronic structure of amorphous germanium. Phys Status Solidi, 1966, 15: 627-637

58 Tsunekawa S, Fukuda T, Kasuya A. Blue shift in ultraviolet absorption spectra of monodisperse $\mathrm{CeO}_{2-x}$ nanoparticles. J Appl Phys, 2000, 87: 1318-1321

59 Flores E, Ares JR, Castellanos-Gomez A, et al. Thermoelectric power of bulk black-phosphorus. Appl Phys Lett, 2015, 106: 022102

60 Ziletti A, Carvalho A, Campbell DK, et al. Oxygen defects in phosphorene. Phys Rev Lett, 2015, 114: 046801

Acknowledgements This work was supported by the National Natural Science Foundation of China (51173097, 91333109, 61434001 and 61574083), the National Key Basic Research Program of China (2013CB632900), Tsinghua University Initiative Scientific Research Program (20131089202) and the Open Research Fund Program of the State Key Laboratory of Low-Dimensional Quantum Physics (KF201516).

Author contributions Yan Q and Ren TL supervised the project. Zhang $\mathrm{Z}$ and Yan Q synthesized the $o$-BP single crystal and performed the structural and optical characterization. Xin X and Zhang Z fabricated the bottom electrode structure FET and carried out the device measurement, analyzed the data, and wrote the manuscript. Li Q helped with the XRD and X-ray rocking curve analysis. Yang Y and Ren TL contributed to the FET measurements and analysis of the data.

Conflict of interest These authors declare that they have no conflict of interest. 

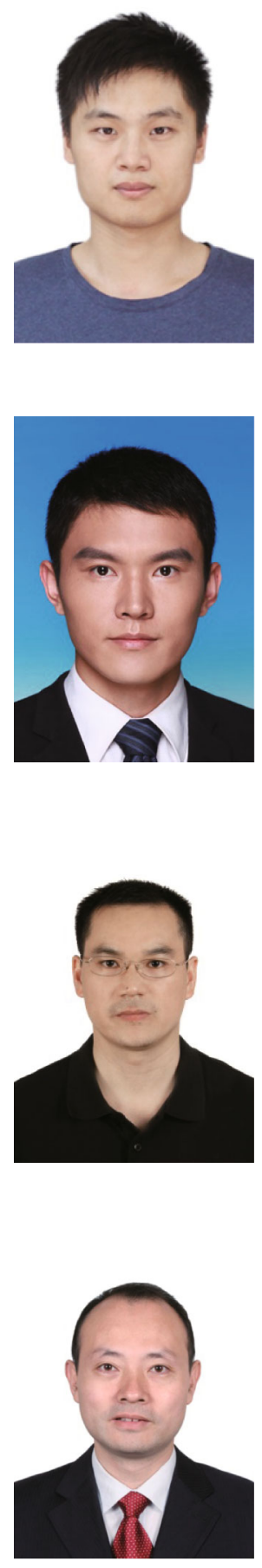

Ziming Zhang is currently a PhD candidate under the supervision of Prof. Qingfeng Yan at the Department of Chemistry, Tsinghua University. His research interests focus on the synthesis and application of black phosphorus-based $2 \mathrm{D}$ materials.

Xin Xin is a master student under the supervision of Prof. Tian-Ling Ren at the Institute of Microelectronics, Tsinghua University. His research interest is novel devices based on 2D materials.

Qingfeng Yan received his PhD degree from the Institute of Semiconductors, Chinese Academy of Sciences in 2003. He joined the Department of Chemical \& Biomolecular Engineering, National University of Singapore as a research fellow in 2003. From 2006, he worked with the School of Materials Science and Engineering, Nanyang Technological University and the Department of Materials Science and Engineering, Massachusetts Institute of Technology as a joint postdoctoral fellow. He joined the Department of Chemistry, Tsinghua University as an associate professor in 2008. His current research interest focuses on synthetic single crystals and chemical self-assembly approach to functional thin films.

Tian-Ling Ren is a full professor of the Institute of Microelectronics, Tsinghua University since 2003. He received his $\mathrm{PhD}$ degree in solid-state physics from the Department of Modern Applied Physics, Tsinghua University, China in 1997. His main research interests include new material based micro/nano devices and systems, flexible electronics, non-volatile memory.

\section{两步加热化学气相传输法合成 3 mm正交相黑磷单晶}

张子明, 辛金垩, 严清峰, 李强, 杨轶, 任天令

摘要 本文采用一种温和的、绿色的两步加热化学气相传输反应方法合成了 3 mm正交相黑磷单晶, 产率高达 $90 \%$. 该反应体系由矿化剂金属 锡和单质碘以及前驱体红磷构成. 反应产物中, 磷化锡是唯一的副产物, 这大大地简化了正交相黑磷单晶后续的分离和纯化过程. 首次运用X射 线摇摆曲线研究了所合成的正交相黑磷单晶, 得到的摇摆曲线的半高峰宽为 21.65 弧秒, 揭示了其优异的单晶质量. 通过一种全干法转移技术制 备了基于机械剥离的少层磷烯的底电极结构的场效应晶体管. 在 $6 \mathrm{~nm}$ 厚的磷烯薄片上构筑的场效应晶体管表现出目前最高纪录的空穴迁移率 $\left(1744 \mathrm{~cm}^{2} \mathrm{~V}^{-1} \mathrm{~s}^{-1}\right)$ 和高的开关比 $\left(10^{4}\right)$, 该结果进一步证明所发展的两步加热化学气相传输反应方法合成的正交相黑磷单晶具有优异的单晶质量. 